\title{
Center for Nanophase Materials Sciences
}

\author{
Linda L. Horton* \\ *Center for Nanophase Materials Sciences, Oak Ridge National Laboratory, Post Office Box 2008, \\ Oak Ridge, TN 37830-6496
}

The Center for Nanophase Materials Sciences (CNMS) is the Department of Energy's newest user facility at Oak Ridge National Laboratory (ORNL). A foundation for the CNMS user program is its location next door to the Spallation Neutron Source (SNS) (see Fig. 1) and its ties to other ORNL user programs, including the National Leadership Computing Facility, the High Flux Isotope Reactor, and the microscopy facilities in the Shared Research Equipment User Program and High Temperature Materials Laboratory.

The CNMS facility is a new 85,000 sq. ft. laboratory and office building that includes a 10,000 sq. $\mathrm{ft}$ nanofabrication clean room facility. Next to and within the clean room, there are low noise (electromagnetic, acoustic, and vibration) rooms for electron-beam equipment, including electron microscopes, focused ion beam instrumentation and electron-beam lithography (see Fig. 2). The ground floor includes a large suite of scanning probes, including both ultrahigh vacuum and ambient environment instruments. Also scheduled for installation on the ground floor is a $700 \mathrm{MHz}$ NMR. The upper levels of the facility are dedicated to synthesis instrumentation and a wide range of standard characterization tools, including those needed for soft materials, x-ray diffraction, and laser-based tools including a tunable micro-Raman system.

Equipment available to CNMS users includes both standard, off-the-shelf, instrumentation and state-of-the-art, unique (or nearly so) tools developed as part of the facility research. One example of the latter group of equipment is a state-of-the-art scanning tunneling microscope being developed as a partnership between ORNL, the University of Tennessee and the University of Houston. This instrument will be equipped for variable temperature down to $300 \mathrm{mK}$ and high magnetic fields up to $9 \mathrm{~T}$.

The CNMS supports research on macromolecular materials, catalysts, functional nanomaterials, and magnetism and transport in nanoscale materials. As a DOE-Basic Energy Sciences (BES) national user facility, research with the CNMS facilities and collaborative support from CNMS staff are freeof-charge for users conducting non-proprietary research that is intended for publication in the open literature. User research proposals are brief and focused-the core of a proposal is a narrative research description of no more than two pages. Selection of the proposals that will get access to the CNMS is based on their scientific and technical quality. The CNMS relies on an external (nonORNL) Proposal Review Committee to evaluate the user proposals and provide ratings that are used to prioritize user access.

Microscopy is one of the most popular tools used in nanoscale science research. CNMS has formed a partnership with the Shared Research Equipment User Facility at ORNL to access analytical and scanning electron microscopes as well as the atom probe field ion microscopes. In addition, under the leadership of Dr. David Joy, CNMS anticipates future research on nanomanipulation for in situ properties assessments in a microscope. ORNL is also part of the DOE-BES TEAM (Transmission Electron Aberration-Corrected Microscope) project to develop the next generation electron 
microscope. This partnership will insure that the right tools are developed to support nanoscale science research for the future.

For more information on the CNMS, visit our website at http://www.cnms.ornl.gov/. The Center for Nanophase Materials Sciences is sponsored by the Office of Basic Energy Sciences, U.S. Department of Energy, under contract DE-AC05-00OR22725 with UT-Battelle, LLC.

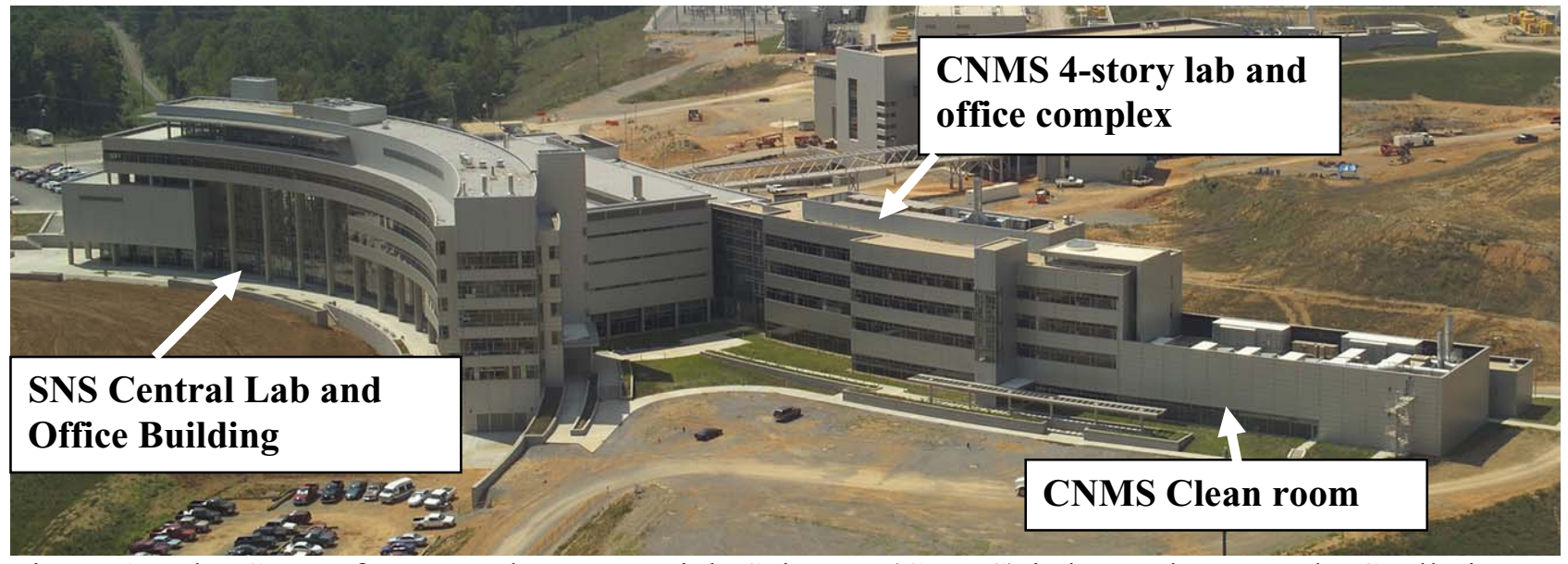

Figure 1: The Center for Nanophase Materials Sciences (CNMS) is located next to the Spallation Neutron Source (SNS) on Oak Ridge National Laboratory’s Chestnut Ridge Campus.

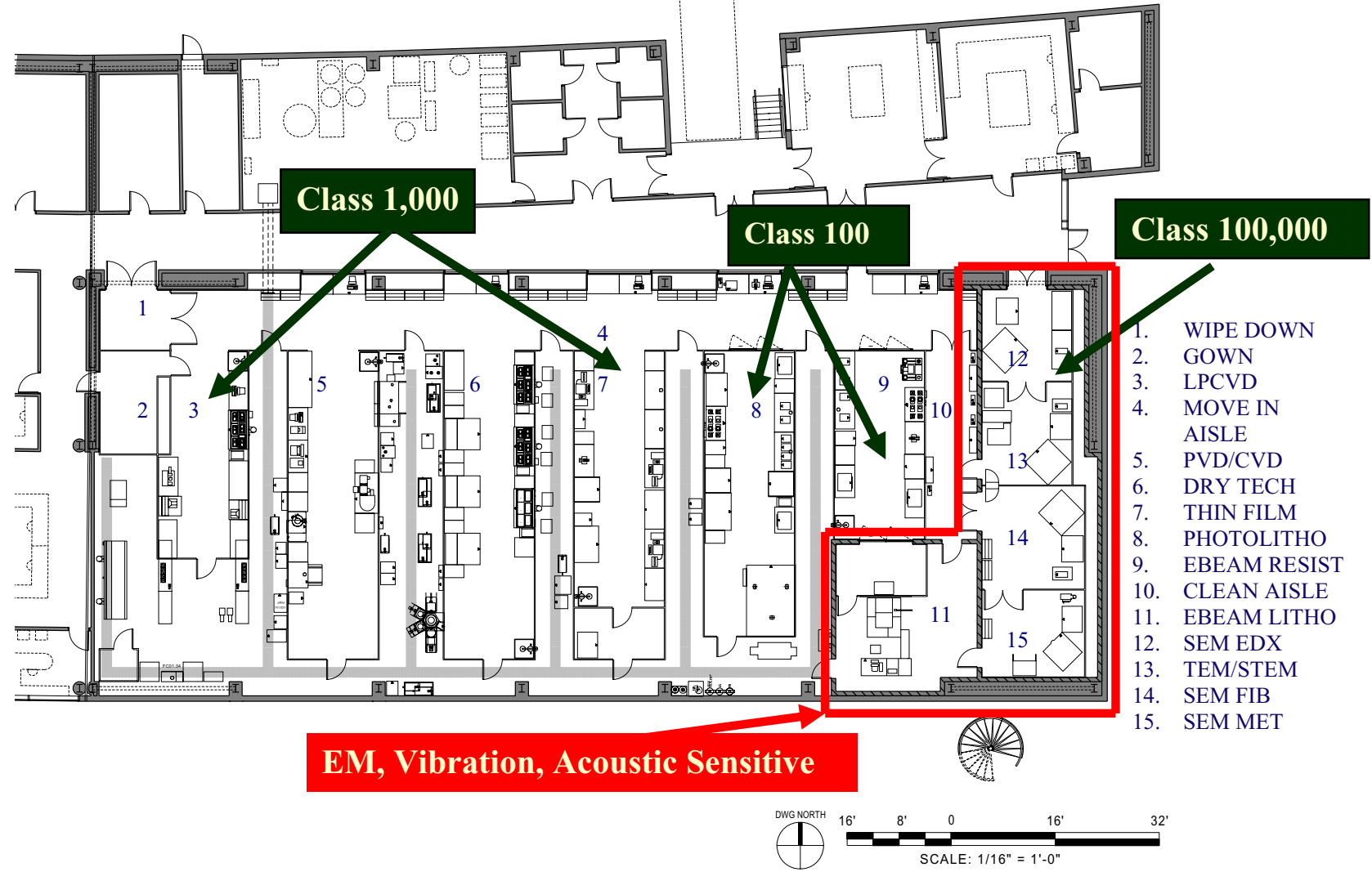

Figure 2: CNMS clean room and electron-beam equipment suite. 